\title{
ESTRATEGIAS DE INTERVENCIÓN PARA DISMINUIR LA VIOLENCIA Y LA DELINCUENCIA: AVANCES Y DESAFÍOS EN REPÚBLICA DOMINICANA
}

\section{Intervention strategies to reduce violence and crime: Progress and challenges in the Dominican Republic}

\section{Magnolia Ortiz Rodríguez}

Facultad de Ciencias Económicas y Sociales de la Universidad Autónoma de Santo Domingo, República Dominicana Correo-e: magnolia_ortiz06@hotmail.com ORCID: 0000-0003-1524-6022

Recibido: 15/1/2019 • Aprobado: 22/6/2019

Cómo citar: Ortiz Rodríguez, M. (2019). Estrategias de intervención para disminuir la violencia y la delincuencia: avances y desafíos en República Dominicana. Ciencia y Sociedad, 44(3), 51-67. https://doi.org/10.22206/cys.2019.v44i3.pp51-67

\section{Resumen}

El objetivo de este estudio es analizar diversas estrategias de prevención de la violencia y la delincuencia, que cuenten con respaldo científico de su eficacia. Se hace una comparación de estos con los programas "Seguridad Democrática" y el "Plan de Seguridad Ciudadana" de República Dominicana. Es un estudio de tipo documental, basado en fuentes secundarias y se utilizan las técnicas de análisis y comparación. Los resultados más importantes revelan que durante la ejecución del Plan Seguridad Democrática, la tasa de homicidios aumentó en un $26 \%$. Sin embargo, durante la ejecución del Plan de Seguridad Ciudadana, la tasa de sobrepoblación penitenciaria ha aumentado en un $22 \%$.

Dentro de los avances del Plan de Seguridad Ciudadana, se destaca la utilización de las TIC. Además, se utilizan los enfoques de persecución del delito y la intervención basada en la comunidad. Los desafíos más importantes son integración de los diferentes sectores en las diversas fases del plan. La utilización del diseño experimental en la evaluación de los programas y verificación de su impacto. Diseño de estrategia en el tercer nivel y disminución de la sobrepoblación penitenciaria.

Palabras clave: medida de seguridad; seguridad ciudadana; estrategia de desarrollo; prevención; violencia; prevención del crimen.

\begin{abstract}
The objective is to analyze violence and crime prevention strategies that have scientific support to be effective. A comparison is made with the Democratic Security programs and the Citizen Security Plan of the Dominican Republic. It is a documentary study that is based on secondary sources. Analysis and comparison techniques are used. The most important results reveal that during the execution of the Democratic Security Plan, the homicide rate increased by $26 \%$. However, it is analyzed that during the execution of the Citizen Security Plan, the prison overpopulation rate has increased by $22 \%$.

Among the advances of the Citizen Security Plan, the use of ICTs stands out. In addition, crime prosecution and community-based intervention approaches are used. The most important challenges are: Integration of the different sectors in the different phases of the Plan, use of the experimental design in the evaluation of the programs and verify their impact, design of strategies in the third level and reduction of the prison overpopulation.
\end{abstract}

Keywords: citizen security; development strategy; prevention; violence; crime prevention. 


\section{Introducción}

Es necesario diferenciar los términos violencia y delito. La violencia se define como:

El uso deliberado de la fuerza física o el poder, ya sea en grado de amenaza o efectivo, contra uno mismo, otra persona o un grupo o comunidad, que cause o tenga muchas probabilidades de causar lesiones, muerte, daños psicológicos, trastornos del desarrollo o privaciones. (Organización Mundial de la Salud, OMS, 2014, p. 2).

El delito se refiere al "conjunto de conductas ilegales que son contrarias a las leyes o normas de un Estado y que trae como consecuencia el castigo o sanción". (Programa de las Naciones Unidas para el Desarrollo, PNUD, 2014, p. 6). La violencia y la delincuencia se diferencian en que se puede cometer delito sin violencia y actos de violencia no considerarse como delito; no obstante, ambas constituyen amenazas a la vida y a la integridad física y patrimonial de las personas.

La alta tasa de violencia y delincuencia trae como resultado la inseguridad ciudadana. En ese sentido, se entiende como inseguridad ciudadana: "situación en la cual el Estado no cumple, total o parcialmente, con su función de brindar protección ante el crimen y la violencia social, lo que significa una grave interrupción de la relación básica entre gobernantes y gobernados. (Comisión Interamericana de Derechos Humanos, CIDH, 2009, p. 9). La inseguridad puede ser objetiva y subjetiva.

La inseguridad objetiva se mide mediante estadísticas de hechos delictivos que se cometen en una comunidad, cuantificando la cantidad de denuncias, el tipo de delitos, datos demográficos de las víctimas y los victimarios. También se realizan encuestas sobre la victimización, que permiten medir la cantidad de víctimas o "cifras ocultas" de personas que no denunciaron. (Instituto Interamericano de Derechos Humanos, IIDH, 2011, p. 25). Por otro lado, la inseguridad subjetiva se establece con base en la percepción de la delincuencia y el miedo a la violencia y al delito de los habitantes. Tanto la inseguridad objetiva y subjetiva pueden medirse a través de encuestas de victimización.

La seguridad ciudadana es una dimensión de la seguridad humana que el Estado debe garantizar, implementando políticas que promuevan el bienestar de las personas. La seguridad ciudadana "Tiene que ver con las obligaciones del Estado como garante de los derechos de quienes se encuentran bajo su jurisdicción" (Instituto Interamericano de Derechos Humanos, IIDH, 2011, p. 21).

En este artículo se muestra la situación de inseguridad de América Latina y El Caribe y, además, se analizan algunas estrategias que han dado resultado en la prevención de la violencia y la delincuencia. De igual modo, se hace una comparación entre las estrategias de prevención que han dado resultado y las políticas de seguridad ciudadana en República Dominicana.

\section{Victimización y percepción de inseguridad en América Latina y el Caribe}

Por varios años se ha mostrado que América es la región más violenta del mundo, "se estima que en 2012 hubo 475,000 muertes por homicidio en todo el mundo y la Región de las Américas, obtuvo la mayor cantidad por cada 100.000 habitantes" (Organización Mundial de la Salud, OMS, 2014, p. 8). Sin embargo, se observa que la tendencia sigue aumentando. "Entre 2000 y 2013, los homicidios se redujeron en todas las regiones del mundo, con la excepción del continente americano, en especial debido a las altas tasas de América Latina y el Caribe" (Vilalta-Perdomo, 2017, p. 5). La proyección para el año 2030 es que todas las regiones del mundo, excepto nuestro continente, tendrán tasas promedio de homicidios por debajo de los cinco homicidios por cada cien mil habitantes. En cambio, en las Américas estaremos sufriendo tasas promedio de homicidios superiores a los treinta homicidios por cada cien mil habitantes. 


\section{Estrategias de intervención para disminuir la violencia y la delincuencia: avances y desafíos en República Dominicana}

En correspondencia con lo anterior, se ha estimado que, dentro de las cincuenta ciudades más violentas del mundo, en el año 2017, cuarenta y seis pertenecen a la región de América Latina y el Caribe, situando a la ciudad de Los Cabos, México, con el número uno, con una tasa de $111.33 \%$ por cada 100.000 habitantes, y la ciudad de Cúcuta, Colombia, en el número 50, con una tasa de $34.78 \%$ por cada 100.000 habitantes. (Seguridad, Justicia y Paz, 2018).

Parecido al ańo anterior, en 2018, de las cincuenta ciudades más violentas del mundo, cuarenta y siete se ubican en el continente americano y cuarenta y dos en América Latina. Entre ellas, se encuentran quince en México, catorce en Brasil, seis en Venezuela, cuatro en Estados Unidos, dos en Colombia, dos en Honduras; y una en Guatemala, El Salvador, Puerto Rico y Jamaica, respectivamente.

Dentro de las ciudades más violentas del mundo, Tijuana, México, ocupó el primer lugar en 2018, con una tasa de $138.5 \%$ por cada 100,000 habitantes; y el estado de New Orleans, Estados Unidos, ocupó el lugar número 50, con el $36.8 \%$ por cada 100,000 habitantes. (Seguridad, Justicia y Paz, 2019)

De acuerdo al Informe de las Naciones Unidas contra la Droga y el Delito, UNODC, 2019, las Américas continúan reportando altas tasas de homicidios, y por varios años, los hombres han tenido más alto riesgo de ser víctima de homicidios. El informe revela que "los hombres jóvenes están especialmente en riesgo, con una tasa para hombres de 18 a 19 años estimada en 46 por 100,000, mucho más alta que el riesgo que enfrentan sus pares en otras regiones. (United Nations Office on Drugs and Crime (UNODC), 2019).

Alrededor del $81 \%$ de las víctimas de homicidios registradas en 2017 eran hombres y nińos, y la tasa global de homicidios para la población masculina es aproximadamente cuatro veces la tasa de mujeres y niñas.

En el caso de las mujeres, el mayor número $(20,000)$ de todas las mujeres asesinadas en todo el mundo por parejas íntimas o familiares en 2017 estuvo en Asia, seguido de África $(19,000)$, América $(8,000)$, Europa $(3,000)$ y Oceanía (300). El estudio revela que América ocupó el tercer lugar de homicidios hacia las mujeres.

La alta tasa de victimización produce una percepción de inseguridad que se traduce en temor a ser víctima. En ese orden, la encuesta de victimización y percepción de inseguridad Latinobarómetro (2016), arrojó que el $36 \%$ de los latinoamericanos reportó haber sido víctima de algún delito; siendo los países con mayor porcentaje de victimización: Venezuela con $48 \%$, México $46 \%$, Argentina $41 \%$ y República Dominicana $41 \%$.

En contraste con la victimización, el $88 \%$ de los ciudadanos latinoamericanos dijo sentir temor a ser víctima de un delito: el $14 \%$ ocasionalmente, el $29 \%$ algunas veces y el $43 \%$ todo o casi todo el tiempo. (Latinobarómetro, 2016, p. 57).

Es importante aclarar que las encuestas de victimización son instrumentos utilizados para medir la cifra oculta de delitos que no se denunciaron, y también miden la percepción de inseguridad de los ciudadanos.

\section{Victimización en República Dominicana}

La República Dominicana ha experimentado una tasa muy alta de violencia durante varios ańos. La tabla a continuación presenta el índice de violencia desde el año 2000 a 2017, según la Oficina Nacional de Estadística ONE.

Tabla 1. Homicidios año 2000-2017

\begin{tabular}{|c|c|c|}
\hline Año & Homicidios & Tasa \\
\hline 2000 & 1,090 & 13 \\
\hline 2001 & 1,086 & 13 \\
\hline 2002 & 1,230 & 14 \\
\hline 2003 & 1,656 & 19 \\
\hline 2004 & 2,323 & 26 \\
\hline 2005 & 2,382 & 26 \\
\hline
\end{tabular}




\begin{tabular}{|c|c|c|}
\hline Año & Homicidios & Tasa \\
\hline 2006 & 2,107 & 23 \\
\hline 2007 & 2,092 & 23 \\
\hline 2008 & 2,357 & 25 \\
\hline 2009 & 2,378 & 25 \\
\hline 2010 & 2,474 & 26 \\
\hline 2011 & 2,517 & 26 \\
\hline 2012 & 2,268 & 23 \\
\hline 2013 & 1,990 & 20 \\
\hline 2014 & 1,810 & 18 \\
\hline 2015 & 1,680 & 17 \\
\hline 2016 & 1,616 & 16 \\
\hline 2017 & 1,561 & 15 \\
\hline
\end{tabular}

Fuente: elaboración propia, basada en la Oficina Nacional de Estadística, ONE.

La tabla anterior refleja que desde el año 2000 la violencia se ha incrementado, teniendo su más alto nivel entre el 2004 y el 2012. Desde 2013 se han registrado niveles más bajos de violencia, pero sigue por encima de la tasa normal. Desde 2004 a 2017, se registra una disminución de la tasa de violencia de un $26 \%$.

Ampliando lo anterior, la Procuraduría General de la República, PGR, muestra que en 2015 hubo un total de 1,675 homicidios en todo el país, con una tasa de $16.78 \%$ homicidios por cada 100.000 habitantes. (Procuraduría General de la República, PGR, 2016). Sin embargo, aunque la tasa de homicidios fue muy alta en 2015, hubo una disminución en relación con el 2014, en el cual se reportó un total de 1,812 casos de homicidios y una tasa de $18.22 \%$ por cada 100.000 habitantes. Estos resultados reflejan 137 casos de homicidios menos en 2015, en relación con el año anterior. En cuanto a las causas de los homicidios, cabe destacar que los homicidios asociados con la delincuencia en 2015 fueron 455 , con una tasa de $4.56 \%$; los no relacionados con la delincuencia fueron 822 y una tasa de $7.8 \%$. Este dato revela que los homicidios por conflictos personales fueron dos veces mayores que los que acompańaban a otro delito. La siguiente tabla muestra esos resultados.
Tabla 2. Homicidios relacionados con la delincuencia en 2015

\begin{tabular}{|l|r|r|}
\hline Caso & Total & Porcentaje \\
\hline Robo o atraco & 366 & $81 \%$ \\
\hline Relacionados con droga & 65 & $14 \%$ \\
\hline Secuestro & 1 & $0 \%$ \\
\hline Servicio policial & 13 & $3 \%$ \\
\hline Sicariato & 10 & $2 \%$ \\
\hline Total & $\mathbf{4 5 5}$ & $\mathbf{1 0 0} \%$ \\
\hline
\end{tabular}

Fuente: elaboración propia, basada en Procuraduría General de la República (PGR, 2016).

De acuerdo a la tabla anterior, el $81 \%$ de los homicidios asociados con delincuencia en 2015 se relacionó con robo o atraco; mientras que el $14 \%$ se vinculó con el delito de drogas. Los homicidios no relacionados con la delincuencia se muestran en la tabla siguiente:

Tabla 3. Homicidios no relacionados con la delincuencia

\begin{tabular}{|l|r|r|}
\hline Causa de homicidio & Cantidad & Porcentaje \\
\hline Accidental & 30 & $4 \%$ \\
\hline Bala perdida & 1 & $0 \%$ \\
\hline Infanticidio & 8 & $1 \%$ \\
\hline Pasional & 13 & $2 \%$ \\
\hline Riña personal & 598 & $73 \%$ \\
\hline Riña en tránsito & 5 & $1 \%$ \\
\hline Violencia intrafamiliar & 17 & $2 \%$ \\
\hline Violencia sexual & 8 & $1 \%$ \\
\hline Feminicidio & 142 & $17 \%$ \\
\hline Total & $\mathbf{8 2 2}$ & $\mathbf{1 0 0} \%$ \\
\hline
\end{tabular}

Fuente: elaboración propia, basada en Procuraduría General de la República (PGR, 2016). 
Los resultados mostrados en la tabla anterior evidencian que el $73 \%$ de los homicidios no relacionados con la delincuencia corresponden a la riña personal, seguida por el feminicidio con un $17 \%$. El $4 \%$ corresponde a homicidio accidental.

Los resultados por provincias en 2017 muestran que hubo una alta tasa de violencia a nivel nacional; excepto la provincia Santiago Rodríguez que tuvo una tasa por debajo del promedio. Se puede apreciar que La Romana obtuvo uno de los niveles más altos de homicidios, con $24 \%$ por cada 100.000 habitantes; una tasa superada por la provincia de Elías Piña y La Altagracia, las cuales tuvieron un $25 \%$ de homicidios por cada 100.000 habitantes. (Procuraduría General de la República, 2017).

Según Latinobarómetro (2016), el $41 \%$ de dominicanos fue víctima de algún delito en 2016. Estos datos corroboran los resultados de la encuesta ENHOGAR, 2015, en la cual se observó que el $29.8 \%$ fue víctima de al menos un acto delictivo durante ese año, y $14.3 \%$ identificó que su vivienda fue objeto de robo o intento de robo; en total, $41.1 \%$ de los dominicanos fue víctima de delincuencia en 2015. (Oficina Nacional de Estadística, 2016).

Los resultados de percepción de inseguridad reflejan que en República Dominicana hubo una alta percepción de inseguridad en el año 2016. En ese orden, la encuesta ENHOGAR refleja que el $33.2 \%$ dijo sentir mucho miedo de ser víctima de delito; el $17.7 \%$ dijo sentir algo de miedo; el $17 \%$ dijo sentir poco miedo a ser víctima. En total, el $69 \%$ de los dominicanos sintieron miedo a ser víctima; mientras que el $31 \%$ dijo no sentir miedo a ser víctima de delitos. (Oficina Nacional de Estadística, 2016).

Por otro lado, los resultados de Latinobarómetro (2016) revelaron que el $86 \%$ de los dominicanos sintió miedo de ser víctima de un delito; $51 \%$ dijo tener miedo a la violencia o a la delincuencia todo o casi todo el tiempo, el $35 \%$ en algún momento ha tenido miedo de ser víctima de la delincuencia y solo el $14 \%$ dijo no sentir miedo de ser víctima.

\section{Condiciones de los privados de libertad}

Las estrategias de prevención deben tener en cuenta las condiciones carcelarias y de respeto de los derechos humanos. En ese sentido, se ha encontrado que América Latina y el Caribe poseen indicadores de violación de los derechos humanos de los privados de libertad, causada, fundamentalmente, por la sobrepoblación penitenciaria. (Comisión Interamericana de Derechos Humanos, 2011). La sobrepoblación penitenciaria se define como:

situación en la que la densidad penitenciaria es mayor que 100 , porque hay más personas privadas de libertad que la capacidad establecida para una prisión o para la totalidad del sistema; cuando la densidad es igual o mayor que 120 , se considera sobrepoblación crítica o hacinamiento crítico. (Carranza, 2012, p. 30).

Las consecuencias del hacinamiento tienen diferentes repercusiones; por ejemplo, la manera infrahumana en que duermen los reclusos:

En algunos centros de reclusión el nivel de hacinamiento puede ser tan agudo que fuerza a los reclusos a dormir por turnos, o encima uno de otro, compartir camas o atarse a las barras de las ventanas para poder dormir de pie. (Naciones Unidas, 2014, p. 15).

La prisión preventiva, que debería ser utilizada como último recurso, sin embargo, se volvió una pena anticipada y sobre utilizada. (Barros Leal, 2014 , p. 105). Se ha señalado que la sobrepoblación carcelaria se debe a lo siguiente:

El uso desmesurado de la medida o la pena privativa de libertad; el rezago judicial de los expedientes de gran parte de la población en reclusión, casi el $50 \%$ son procesados y la falta de utilización de penas alternativas o sustitutivas de la pena privativa de libertad. (Comisión Nacional de los Derechos Humanos, 2015, p. 3). 
La República Dominicana registra un incremento en las condiciones de privación de libertad. A continuación, se muestra la cantidad de privados de libertad desde 2008 a 2018.

Tabla 4. Privados de libertad en República Dominicana, 2008-2018

\begin{tabular}{|c|c|}
\hline Ańo & Privados de libertad \\
\hline 2008 & 16,718 \\
\hline 2009 & 19,151 \\
\hline 2010 & 20,743 \\
\hline 2011 & 21,935 \\
\hline 2012 & 24,044 \\
\hline 2013 & 25,428 \\
\hline 2014 & 25,203 \\
\hline 2015 & 24,617 \\
\hline 2016 & 25,437 \\
\hline 2017 & 26,782 \\
\hline 2018 & 26,078 \\
\hline
\end{tabular}

Fuente: elaboración propia, basada en Oficina Nacional de Estadística ONE.

Tal como lo muestra la tabla, la cantidad de privados de libertad ha aumentado cada año; en 2008 la cantidad de privados de libertad era casi 17,000; en 2018 la cifra subió a más de 26,000 privados de libertad. Esto quiere decir que, de 2008 a 2018, la cantidad de personas privadas de libertad aumentó en un $22 \%$. En cuanto a las condiciones jurídicas de los privados de libertad, se evidencia que alrededor del $65 \%$ de los presos están en condiciones preventivas, y solo el $35 \%$ condenado.

La alta población penitenciaria trae como consecuencias el hacinamiento, problemas de salud y un alto costo al Estado. Las alternativas a la prisión preventiva representan importantes ventajas en distintos sentidos. En primer lugar, la aplicación de las mismas constituye una de las medidas más eficaces para: a) evitar la desintegración y estigmatización comunitarias derivadas de las consecuencias personales, familiares y sociales que genera la prisión preventiva; b) disminuir las tasas de reincidencia, y c) utilizar de manera más eficiente los recursos públicos. (Comisión Interamericana de Derechos Humanos, 2017, p. 79).

\section{Enfoques para enfrentar la violencia y la delin- cuencia en América Latina y el Caribe.}

Para prevenir la violencia y la delincuencia en América Latina y el Caribe se han utilizado diferentes enfoques. Dentro de ellos está el enfoque sálvese quien pueda, se caracteriza por una actitud de indiferencia y negación por parte de los organismos públicos frente al problema de la violencia y el delito. Su enfoque atrasó la incorporación del tema a la agenda pública y, en consecuencia, se promovieron soluciones de naturaleza privada e individual. Bajo este paradigma se evidenció un crecimiento vertiginoso de los servicios de seguridad privada, del armamentismo civil y un cambio acelerado en el perfil urbanístico. (Chinchilla \& Vorndran, 2018, p. 12).

El enfoque mano dura "aboga por la vigilancia policial punitiva y políticas orientadas a la justicia criminal y penal, e incluyen la participación de agentes militares y de mantenimiento del orden". (Aguirre \& Muggah, 2017, p. 26). De acuerdo a esta perspectiva, el delito disminuye con la represión y el castigo. Es importante resaltar que, para que este enfoque sea efectivo, deben considerarse la eficacia de la policía, el sistema judicial y el sistema penitenciario; y garantizar lo siguiente:

Un servicio de policía basado en la eficiencia: que despliegue una actividad preventiva y de atención directa ante las necesidades de la comunidad, la mitigación del crimen, otorgando la detección y la captura del delincuente o criminal.

La aplicación de un efectivo sistema de justicia: con acceso equitativo y amplio, que ofrezca garantías para su aplicación, y disuada la acción delincuencial con una real aplicación de penas. 


\section{Estrategias de intervención para disminuir la violencia y la delincuencia: avances y desafíos en República Dominicana}

Una infraestructura acorde para el servicio penitenciario: que otorgue garantías y condiciones para el cumplimiento de condenas, motive y promueva la resocialización e impida la continuidad de la participación delictiva y criminal desde los centros penitenciarios (Organización de Estados Americanos, OEA, 2012).

En otro orden, el enfoque de las causas estructurales "enfatizan la prevención primaria, secundaria y terciaria, junto con intervenciones más integrales que combinan el mantenimiento del orden con estrategias de bienestar social centradas en la comunidad". (Aguirre \& Muggah, 2017). En el nivel primario, la prevención del delito y la victimización deben establecerse basadas en las políticas donde se garantice el acceso a los servicios básicos de las comunidades, disminuyendo los riesgos y la vulnerabilidad que conducen a conductas delictivas:

La acción preventiva conjuga la responsabilidad del Estado en la formulación de políticas de inversión social en educación, empleo y salubridad, para desestimular focos de oferta para el crimen, fundamentadas en la infancia y adolescencia. (Organización de Estados Americanos, OEA, 2012).

Las políticas públicas deben estar basadas en prevenir la violencia y la delincuencia utilizando estrategias en los tres niveles. (Sistema de Integración Centroamericana, SICA, 2014, p. 9).

En otro orden, las intervenciones basadas en evidencia son aquellas para las cuales la comunidad científica cuenta con estudios experimentales o cuasi experimentales que demuestran los efectos positivos de estas. Es decir, los estudios experimentales o cuasi experimentales generan evidencia sobre el nivel de eficacia de una determinada intervención, en términos del nivel de logro de los objetivos con que se diseñó su modelo. (Tocornal et al., 2011, p. 9).

\section{Intervenciones basadas en evidencia científica}

A continuación, se exponen algunas estrategias, las cuales se han evaluado bajo evidencia científica de que han dado resultados.

Prevención de la delincuencia mediante el diseño ambiental: esta metodología no solo se orienta a los aspectos tradicionales de la prevención situacional, sino que aborda también como componentes centrales la reducción del temor al delito y el aumento de la cohesión comunitaria. Dentro de esta metodología se pueden identificar las siguientes estrategias:

Comunidades que cuidan: Communities that Care (CTC) es un programa de prevención comunitaria basado en la coalición, que tiene como objetivo prevenir las conductas problemáticas de los jóvenes, incluidos el consumo de alcohol por menores de edad, el uso de tabaco, la violencia, la delincuencia, el abandono escolar y el abuso de sustancias. CTC trabaja a través de una junta comunitaria para evaluar los factores de riesgo y protección entre los jóvenes en su comunidad, mediante una encuesta de población basada en la población. La junta trabaja para implementar programas probados y efectivos para abordar los problemas y las necesidades que se identifican. (Instituto de Políticas Públicas del Estado de Washington, WSIPP, 2017). La tabla siguiente muestra el costo y los beneficios de este programa.

Tabla 5. Costo-beneficio del programa Comunidades que cuidan

\begin{tabular}{|c|c|c|c|}
\hline Nombre del programa & $\begin{array}{c}\text { Costo neto del programa por } \\
\text { participante }\end{array}$ & Beneficios menos costos & $\begin{array}{c}\text { Oportunidad: el programa producirá } \\
\text { beneficios mayores que los costos }\end{array}$ \\
\hline Comunidades que cuidan & $\$ 593$ & $\$ 2,555$ & $82 \%$ \\
\hline
\end{tabular}

Fuente: elaboración propia, con base en Instituto de Políticas Públicas del Estado de Washington, WSIPP, 2017. 
Como muestra la tabla, el costo por participante en el programa Comunidades que cuidan tuvo un costo de $\$ 593$ por participante, un beneficio de $\$ 2,555$ y cuenta con un $82 \%$ de que los beneficios sean mayores que los costos.

\section{Estrategia basada en la comunidad}

La Agencia de los Estados Unidos para el Desarrollo Internacional, USAID, elaboró una estrategia de prevención de la violencia y la delincuencia en tres países de América Central. En dicho proyecto se utilizó la estrategia basada en la comunidad, que incluye actividades como la planificación de los comités a nivel municipal; observatorios de delincuencia y recolección de datos; prevención de la delincuencia a través de un modelo ambiental (tales como una mejora en el alumbrado público, borrado de grafitis, limpieza de espacios públicos); programas para los jóvenes en situación de riesgo (tales como centros de alcance, capacitación profesional, programas de mentores); y policía comunitaria. Los proyectos de prevención de la delincuencia de USAID basados en la comunidad son intrínsecamente intersectoriales. Es decir, integran intervenciones en educación y capacitación profesional, desarrollo económico y empleo, salud pública y gobernanza. (Berk-Seligson, Orcés, Pizzolitto, Seligson, \& Wilson, 2014).

Los resultados del proyecto de prevención de la USAID reflejaron que hubo una reducción de los reportes de asesinatos en $51 \%$; reducción en los reportes de venta de drogas ilegales en $36 \%$; reducción en los reportes de robos en $19 \%$. (Berk-Seligson, Orcés, Pizzolitto, Seligson, \& Wilson, 2014).

En el caso de Chile, el programa Barrio en Paz Residencial tuvo un impacto del 29,6\% en la disminución de la victimización en hogares. El Programa de Seguridad Ciudadana que ejecuta Trinidad y Tobago, y concentra parte importante de sus actividades en veinte y dos comunidades, seleccionadas por experimentar una alta incidencia delictual, lleva a cabo diversos programas de intervención, tales como creación de clubes para jóvenes, mantenimiento de espacios públicos, atención sicológica para víctimas y desarrollo de programas de mediación de conflictos. En las comunidades intervenidas se constata una reducción de un $55 \%$ en los homicidios entre el 2008 y el 2012. (Frühling, 2012, p. 41).

\section{Estrategias basadas en la escuela}

Demasiado bueno para las drogas: es un programa de prevención basado en la escuela para estudiantes de $\mathrm{K}-12$. Está diseñado para aumentar las competencias sociales y disminuir los factores de riesgo asociados con el consumo de alcohol, tabaco y otras drogas. El programa consta de diez lecciones interactivas en el aula adaptadas para diferentes niveles de grado. (Instituto de Políticas Públicas del Estado de Washington, WSIPP, 2017). La tabla siguiente muestra los costos y beneficios de este programa.

Tabla 6. Costo beneficio del programa Demasiado bueno para las drogas

\begin{tabular}{|c|c|c|c|}
\hline Nombre del programa & $\begin{array}{c}\text { Costo neto del programa por } \\
\text { participante }\end{array}$ & Beneficios menos costos & $\begin{array}{c}\text { Oportunidad: el programa } \\
\text { producirá beneficios mayores } \\
\text { que los costos }\end{array}$ \\
\hline $\begin{array}{c}\text { Demasiado bueno para las } \\
\text { drogas }\end{array}$ & $\$ 54$ & $\$ 443$ & $90 \%$ \\
\hline
\end{tabular}

Fuente: elaboración propia, con base en Instituto de Políticas Públicas del Estado de Washington, WSIPP, 2017.

58 | Ciencia y Sociedad 2019; 44(3): 51-67 • Una Mirada desde lo Particular 


\section{Estrategias de intervención para disminuir la violencia y la delincuencia: avances y desafíos en República Dominicana}

Se muestra en la tabla que el costo por estudiante del programa fue de $\$ 54$; el beneficio de $\$ 443$ y $90 \%$ de posibilidades de que el programa producirá mayores beneficios que costos. Este programa se entrega típicamente en un solo año escolar. Los costos incluyen la capacitación docente, el programa y los materiales del aula, tales como cuadernos de ejercicios, carteles, encuestas, juegos, CD y DVD, y el tiempo del docente para impartir las lecciones, repartidos entre la cantidad de nińos en un aula típica de Washington.

Programa "Aulas en Paz": ejecutado por el Organismo No Gubernamental Convivencia Productiva y la Universidad de Los Andes de Colombia. Se trata de un modelo de intervención que promueve competencias ciudadanas como herramientas para romper el ciclo de la violencia y construir una cultura de paz. Desarrollado a nivel piloto, en forma gradual, se extendió a veintisiete instituciones educativas públicas, de quince municipios del país y afectados por la violencia. Antes del inicio de la implementación en las aulas (pre-test) y al finalizar el primer ańo escolar (post-test), tanto los estudiantes del grupo de control como los del grupo experimental llenaron un cuestionario que mide los niveles de agresión, empatía, asertividad y creencias que legi- timan la agresión. Los docentes también respondieron cuestionarios sobre la agresión y los comportamientos prosociales de los estudiantes. Los resultados mostraron impactos significativos del programa, ya que tanto estudiantes como docentes reportaron consistentemente una disminución en agresión entre quienes recibieron el programa, en contraste con una disminución significativamente menor entre quienes no lo recibieron. (Frühling, 2012, p. 41).

\section{Estrategias coercitivas}

Implementar un oficial de policía adicional con estrategias de puntos calientes: este amplio grupo de estudios estima la efectividad de la vigilancia policial de los puntos calientes (en comparación con las prácticas promedio de todo el estado), principalmente en las jurisdicciones urbanas de los Estados Unidos. La vigilancia policial de los puntos calientes concentra la vigilancia policial en áreas de alta criminalidad o en delitos específicos como el tráfico de drogas. Esta estrategia difiere de la vigilancia policial "tradicional", que generalmente se basa en una patrulla preventiva aleatoria o en una respuesta a las llamadas de servicio. La tabla siguiente muestra los costos y beneficios de este programa.

Tabla 7. Costo-beneficio de Implementar un oficial con estrategias de puntos calientes

\begin{tabular}{|c|c|c|c|}
\hline Nombre del programa & $\begin{array}{c}\text { Costo neto del programa } \\
\text { por participante }\end{array}$ & $\begin{array}{c}\text { Beneficios menos } \\
\text { costos }\end{array}$ & $\begin{array}{c}\text { Oportunidad: el programa } \\
\text { producirá beneficios mayores } \\
\text { que los costos }\end{array}$ \\
\hline $\begin{array}{c}\text { Implementar un oficial de policía } \\
\text { adicional con estrategias de puntos } \\
\text { calientes }\end{array}$ & $\$ 96,637$ & $\$ 421,768$ & $100 \%$ \\
\hline
\end{tabular}

Fuente: elaboración propia. (Instituto de Políticas Públicas del Estado de Washington, WSIPP, 2017). 
La tabla anterior muestra que el costo de implementar un oficial policial en puntos calientes fue de $\$ 96,637$; el beneficio, $\$ 421,768$ y un $100 \%$ de posibilidades de que los beneficios excedan el costo.

La estrategia de implementar un oficial en puntos calientes se ha utilizado en los países de Chile, Colombia y Ecuador, entre otros. Ello revela que el intercambio de experiencias entre los cuerpos policiales es muy activo, aunque a la vez cada uno de los países ha diseñado los planes respectivos de acuerdo a condiciones que les son propias. Se pudo evaluar que la estrategia ha tenido resultados en la reducción de los delitos: en los homicidios esta reducción fue de $16 \%$; en el caso de lesiones personales fue de $9 \%$ y en el hurto a vehículos la reducción que se dio fue de un $22 \%$. (Frühling, 2012, p. 44).

\section{Estrategias basadas en prevención terciaria}

Terapia familiar funcional (FFT) para jóvenes en instituciones estatales: es una intervención estructurada basada en la familia que utiliza un enfoque de varios pasos para mejorar los factores de protección y reducir los factores de riesgo en la familia. Los cinco componentes principales de FFT incluyen 1) compromiso, 2) motivación, 3) identificación de patrones de interacción dentro de la familia, 4) cambio de comportamiento y 5) generalización de interacciones positivas a situaciones nuevas.

Tabla 8. Costo-beneficio del programa Terapia Familiar Funcional

\begin{tabular}{|c|c|c|c|}
\hline Nombre del programa & $\begin{array}{c}\text { Costo neto del progra- } \\
\text { ma por participante }\end{array}$ & $\begin{array}{c}\text { Beneficios menos } \\
\text { costos }\end{array}$ & $\begin{array}{c}\text { Oportunidad: el programa producirá } \\
\text { beneficios mayores que los costos }\end{array}$ \\
\hline $\begin{array}{c}\text { Terapia Familiar Funcional (FFT) } \\
\text { para jóvenes en instituciones estatales. }\end{array}$ & $\$ 3,469$ & $\$ 36,737$ & $96 \%$ \\
\hline
\end{tabular}

Fuente: elaboración propia, con base en Instituto de Políticas Públicas del Estado de Washington, WSIPP, 2017.

La tabla anterior muestra que el costo de Terapia Familiar Funcional tuvo un costo de \$3,469; el beneficio, \$36,737 y un $96 \%$ de posibilidades de que los beneficios excedan el costo.

Desde 2012, Chile está implementando el programa "PAIF 24 Horas". Semanalmente, la policía remite a las municipalidades una lista de niños y adolescentes detenidos o conducidos a unidades policiales. Las víctimas de vulneraciones de derechos son llevadas a la Oficina Municipal de Protección de Derechos y se moviliza a la red de servicios de protección de la infancia. Los casos ingresados por conductas transgresoras de la ley son remitidos a un equipo especializado, que aplica un breve instrumento de valoración de riesgo socio-delictivo para estimar empíricamente la probabilidad de reincidir. Se invita a la familia del niño, niña o adolescente a participar de un servicio de atención cuya intensidad es proporcional al nivel de riesgo. Encontraron reducciones de la reincidencia estadísticamente significativas de 6 puntos porcentuales después de un año de seguimiento. También se observaron reducciones de 6,5 a 13,4 puntos porcentuales para el 


\section{Estrategias de intervención para disminuir la violencia y la delincuencia: avances y desafíos en República Dominicana}

subgrupo de más alto riesgo, y de entre 10,5 y 14 puntos porcentuales para los jóvenes de entre 16 y 18 años.

\section{Uso de las Tecnologías de la Información}

Monitoreo electrónico: esta estrategia implica el uso de unidades de radiofrecuencia o sistema de posicionamiento global (GPS) para monitorear la ubicación de una persona. El monitoreo electrónico se usa para hacer cumplir los requisitos de que una persona permanezca en el hogar, excepto para las actividades aprobadas, como el trabajo, la escuela o el tratamiento. Este metanálisis incluye estudios en individuos que estaban en libertad condicional con monitoreo electrónico. Fueron comparados con individuos similares que recibieron supervisión intensiva, libertad condicional, continuación de la sentencia o confinamiento en el hogar sin supervisión electrónica. (Instituto de Políticas Públicas del Estado de Washington, WSIPP, 2017).

El uso de un dispositivo electrónico disminuye el costo en el sistema penitenciario. El problema principal en América Latina y el Caribe es que la persona acusada debe costear su uso y, como consecuencia, es de difícil el acceso a esta medida por parte de personas en situación de pobreza o con bajos ingresos.

Sistemas Big Data: en seguridad ciudadana, se trataría de la integración de bases de datos sobre reportes policiales, llamadas de emergencia, expedientes judiciales, expedientes carcelarios, redes sociales e Internet, información de CCTV, datos biométricos, y/u otros registros administrativos como el registro vehicular, información tributaria, del seguro social, etc. Esta integración de información en formatos de texto, audio y video, requiere de una gran capacidad de cómputo y un proceso de datificación. El uso de sistemas Big Data para la prevención de la violencia y la delincuencia tiene varios retos. En labores preventivas, los mayores son: la dispersión de fuentes, el volumen y la variedad de la información disponible. (Vilalta-Perdomo, 2017, p. 13).
Desafíos de los programas de intervención en América Latina y el Caribe

A pesar de que los países de América Latina y el Caribe han avanzado en el desarrollo de programas de prevención, quedan muchos desafíos. A continuación, se presenta una lista de los principales desafíos presentados por Chinchilla y Vorndran, 2018:

La integridad. Estrategias articuladas que promuevan una visión compartida del problema y de los principios orientadores, aunque cada actor tenga capacidades e intervenciones diferenciadas. La incorporación de diversos organismos y sectores a la gestión de la seguridad. Necesidad de diseñar estructuras organizativas que faciliten la coordinación y la cohesión interinstitucional.

Multisectorialidad. Estructuras organizativas que faciliten la coordinación y la cohesión interinstitucional entre autoridades nacionales, estatales y municipales. Organización y capacitación de la sociedad civil e integración del sector privado. La creación de consejos para la seguridad y la prevención del delito es una de las respuestas más frecuentes al desafío de la multisectorialidad; sin embargo, se observan problemas y limitaciones asociados a la excesiva burocratización y la ausencia de mecanismos rigurosos para el monitoreo de acciones y el cumplimiento de metas. Además, los lineamientos no siempre permean los niveles operativos de las instituciones, identificándose serios problemas de acoplamiento. Uno de los aspectos más destacados de los nuevos enfoques de la seguridad es el relativo a la necesidad de diseñar e impulsar políticas públicas basadas en evidencia.

Rigurosidad. Esta exigencia supone al menos cumplir con tres condiciones: 1 . disponibilidad y gestión de información fiable y oportuna, 2. existencia de un recurso humano con capacidades técnicas para gestionar políticas asociadas al nuevo paradigma de la seguridad, tanto en las instituciones como en la sociedad civil, y 3. acceso a las tecnologías de la información y la comunicación (TIC) que faciliten la gestión de información para el diseño, implementación y 
monitoreo de las políticas y programas. Los desafíos que enfrenta la rigurosidad son: la disponibilidad y calidad de la información. El abordaje multicausal de la seguridad implica la consideración de un amplio número de variables en todas las fases del proceso de toma de decisiones y, por consiguiente, un personal con capacidades técnicas y profesionales específicas, y competencias transversales - como capacidad para trabajar en equipo, actitud proactiva y resolutiva-, y habilidades para comunicar de manera horizontal y vertical. Las aplicaciones más frecuentes de las TIC tienen que ver con sistemas de georreferenciación, videovigilancia y biométricos, que ya han sido incorporados en la gestión de la seguridad en diversos países.

Sostenibilidad y escalabilidad. Efectivo liderazgo de la sociedad civil. Mecanismos de financiamiento permanentes. Diseño y planificación, evitando la trampa de la experiencia piloto. Algunos de los proyectos que se vienen ejecutando enfrentan dificultades para superar la prueba del tiempo, limitando su vigencia al plazo de financiación por parte de una agencia de cooperación internacional, al período de nombramiento de un director de programa, o al ciclo electoral de gobiernos locales o nacionales (Chinchilla \& Vorndran, 2018).

\section{Estrategias de prevención en República Domi- nicana}

En esta sección se presentan dos programas de seguridad ciudadana en República Dominicana.

El Plan de Seguridad Democrática (PSD). Fue uno de los principales proyectos de nación, enfocado en brindar a la población el acceso a sus derechos fundamentales, principalmente a las personas residentes en zonas marginadas. Este proyecto tuvo sus inicios en el año 2004 y comenzó a desarrollarse plenamente en el 2005, apuntando a la prevención del crimen y la delincuencia callejera, y a combatir las causas que generan inseguridad. Dicho plan tuvo varios programas, dentro de estos:
Programa Barrio Seguro. Los subprogramas fueron: "Financiando Microempresarios de mi Barrio". "Un Techo para mi Barrio". "Becas para mi Barrio". "Centros de Capacitación Tecnológico y Comunal". "Competidores de mi Barrio". "Fiesta de mi Barrio". "Bandas de Música de mi Barrio". "Los Muralistas y Pintores de mi Barrio". "Niños, Niñas y Adolescentes no Escolarizados de mi Barrio".

Estos subprogramas ofrecieron oportunidades de microcréditos a personas emprendedoras para que participaran en los procesos productivos; asegura el acceso a la salud, con centros de atención primaria; gestiona soluciones alimentarias y habitacionales; habilita centros de enseńanza, promueve eventos culturales, artísticos, musicales, deportivos y de recreación; refuerza los valores de la patria y de la sociedad. También recupera el liderazgo social, atiende la escolarización y facilita los estudios superiores.

\section{Programa Municipio Seguro}

Villa de la Seguridad Ciudadana. Red Alimentaria. Protección a Víctimas de la Violencia. Prevención y Seguridad. Control de Bebidas Alcohólicas (COBA). Control de Armas de Fuego (COFA). Control y Regulación de Productos Pirotécnicos. Asistencia en Carretera (ACARREA). Call Center hacia el 9-1-1. Programa Migratorio de Regularización de Extranjeros. Naturalizaciones. Mesa Interinstitucional de Coordinación y Estrategias contra el Microtráfico de Drogas. Ordenamiento de los Cuerpos de Bomberos. El programa se realizó en 115 sectores: treinta y un municipios de la provincia de Santiago; tres en municipios de la provincia Altagracia; dos municipios de la provincia de San Cristóbal y los demás en sectores de Santo Domingo.

\section{Plan Nacional de Seguridad Ciudadana}

Es el plan actual sobre seguridad ciudadana. Busca reducir los niveles de delincuencia en el país. El plan se sustenta sobre dos pilares fundamentales: persecución y prevención del delito. Este plan inició con la participación de más 2,000 efectivos policiales y militares, quienes patrullaban día y noche las calles, 


\section{Estrategias de intervención para disminuir la violencia y la delincuencia: avances y desafíos en República Dominicana}

con el objetivo de proteger a la ciudadanía. Más de 5,000 policías y militares fueron diseminados a nivel nacional para proteger las vidas y los intereses de los ciudadanos. (Dirección de Información, Análisis y Programación Estratégica (DIAPE), 2016). Los subprogramas son los siguientes:

Tabla 9. Subprogramas del Programa Seguridad Ciudadana

\begin{tabular}{|c|c|c|}
\hline & Descripción & Evaluación \\
\hline $\begin{array}{l}\text { Reforma Institucional de } \\
\text { la Policía Nacional. }\end{array}$ & $\begin{array}{l}\text { Capacitación, aumento salarial, registro en sistema de salud de los agentes } \\
\text { policiales. }\end{array}$ & \\
\hline $\begin{array}{l}\text { Implementación del } \\
\text { Sistema Integrado de } \\
\text { Emergencias } 911 .\end{array}$ & $\begin{array}{l}\text { Este servicio supone una revolución en la atención a las emergencias en el } \\
\text { país: rápido, eficiente, moderno, y para todos. }\end{array}$ & $\begin{array}{l}\text { Se han atendido más de } 223,788 \\
\text { llamadas. } \\
\text { La satisfacción de los usuarios del } \\
\text { servicio } 911 \text { es del } 91 \% . \\
\text { Instalación de } 1,300 \text { videocámaras } \\
\text { de vigilancia en el Gran Santo } \\
\text { Domingo. }\end{array}$ \\
\hline $\begin{array}{l}\text { Registro de celulares para } \\
\text { el funcionamiento del } \\
911 \text { y la seguridad. }\end{array}$ & $\begin{array}{l}\text { El registro obligatorio de los números de celulares y prohibió de la venta en } \\
\text { las calles de chips para móviles. }\end{array}$ & \\
\hline $\begin{array}{l}\text { Creación del Consejo } \\
\text { Nacional de Seguridad } \\
\text { Ciudadana. }\end{array}$ & $\begin{array}{c}\text { Encabezado por el Primer Mandatario e integrado por los Ministros de la } \\
\text { Presidencia, de Interior y Policía, quien lo coordina, y de las Fuerzas } \\
\text { Armadas. Además, el Procurador General de la República, el Jefe de la } \\
\text { Policía Nacional, el Presidente del Consejo Nacional de Drogas, el Director } \\
\text { de Control de Drogas y el Asesor del Poder Ejecutivo para el Programa de } \\
\text { Luchas contra el Narcotráfico. }\end{array}$ & \\
\hline $\begin{array}{l}\text { Lanzamiento del } \\
\text { Observatorio de } \\
\text { Seguridad Ciudadana. }\end{array}$ & $\begin{array}{l}\text { El objetivo del Observatorio de Seguridad Ciudadana es recabar, consolidar, } \\
\text { procesar y analizar las informaciones delictuales del país, para orientar y } \\
\text { apoyar acciones y políticas de prevención, reducción y control de la crimina- } \\
\text { lidad. Encabezado por el Ministerio de Interior y Policía. }\end{array}$ & \\
\hline $\begin{array}{l}\text { Creación de la Mesa } \\
\text { Nacional sobre Segu- } \\
\text { ridad, Ciudadanía y } \\
\quad \text { Género. }\end{array}$ & $\begin{array}{c}\text { La Mesa Nacional está compuesta por las instituciones del Gobierno } \\
\text { Central, Gobiernos Intermedios, Gobiernos Locales y la Sociedad Civil } \\
\text { Organizada. El Ministerio de Interior y Policía es el encargado de coordinar } \\
\text { la Mesa Nacional y las Mesas Locales de Seguridad, a fin de garantizar la } \\
\text { interacción e intervención en acciones de prevención y fortalecimiento de la } \\
\text { Seguridad Ciudadana. }\end{array}$ & \\
\hline $\begin{array}{l}\text { Programa de Protección } \\
\text { y Asistencia Vial. }\end{array}$ & $\begin{array}{l}\text { Ese programa incluye un sistema de vigilancia, protección, seguridad y } \\
\text { asistencia vial a nivel nacional, completamente gratis, los siete días de la } \\
\text { semana y las } 24 \text { horas del día. }\end{array}$ & $\begin{array}{l}\text { Más de 250,000 asistencias en las } \\
\text { carreteras del país. }\end{array}$ \\
\hline $\begin{array}{l}\text { Programa de prevención } \\
\text { Vivir Tranquilo }\end{array}$ & $\begin{array}{l}\text { Implica la interacción con todas las organizaciones de la sociedad civil } \\
\text { que operan en los barrios y que participan en él, especialmente las iglesias, } \\
\text { ONGs, junta de vecinos y las asociaciones de padres y amigos de la escuela } \\
\text { que están registradas en el Ministerio de Interior y Policía. }\end{array}$ & $\begin{array}{l}\text { 5,000 jóvenes y ha habilitado } 79 \\
\text { Centros Tecnológicos y Comunales. }\end{array}$ \\
\hline $\begin{array}{l}\text { Estrategia para el control } \\
\text { de drogas }\end{array}$ & $\begin{array}{l}\text { Intensificar las acciones e incrementar los recursos sobre los dos aspectos } \\
\text { fundamentales del problema: el narcotráfico internacional y el } \\
\text { narcomenudeo o microtráfico. }\end{array}$ & $\begin{array}{l}\text { En } 2013 \text { la Dirección Nacional de } \\
\text { Control de Drogas (DNCD) } \\
\text { decomisó 10,664 kilos de diferentes } \\
\text { drogas narcóticas. }\end{array}$ \\
\hline
\end{tabular}

Fuente: elaboración propia, basado en datos de la Dirección de Información, Análisis y Programación Estratégica (DIAPE), 2016. 
Los subprogramas del Plan de Seguridad Ciudadana presentan enfoque de persecución del delito, uso de sistemas de información; tiene una estrategia basada en la comunidad.

\section{Discusión}

\section{Evaluación del Programa Barrio Seguro}

Estuvo orientado mediante el diseño ambiental. Según la literatura, esta metodología ha dado resultados en Estados Unidos y en países de América Latina. Dentro de los hallazgos están: disminución del uso de tabaco en los jóvenes, disminución de la violencia, la delincuencia, prevención del abandono escolar y del abuso de sustancias (Instituto de Políticas Públicas del Estado de Washington, WSIPP, 2017).

Los resultados del proyecto de prevención basado en la comunidad, implementado por USAID en comunidades de tres países de América Central, reflejaron que hubo una reducción de los reportes de asesinatos en $51 \%$; reducción en los reportes de venta de drogas ilegales en $36 \%$; reducción en los reportes de robos en $19 \%$. (Berk-Seligson, Orcés, Pizzolitto, Seligson, \& Wilson, 2014). En ese mismo orden, el Programa Barrio en Paz Residencial tuvo un impacto del 29,6\% en la disminución de la victimización en hogares. En el Programa de Seguridad Ciudadana que ejecuta Trinidad y Tobago en las comunidades intervenidas, se constata una reducción de un $55 \%$ en los homicidios entre el 2008 y el 2012.

El Programa Barrio Seguro, tiene escasa información científica sobre la efectividad en los sectores donde el proyecto fue implementado. Sin embargo, de acuerdo a la estadística, durante el periodo 2004 a 2012, la tasa de violencia fue más alta que en los demás años; con tasa de homicidios de $26 \%$ y 23 $\%$ por cada 100,000 habitantes, lo que manifiesta que la violencia aumentó durante ese periodo.

\section{Plan Seguridad ciudadana}

a. Utilización de sistemas de las TIC: cámaras digitales, sistema integrado 911, Observatorio de Seguridad
Ciudadana para la captura y análisis de información de la violencia y la delincuencia. Las TIC que facilitan la gestión de información para el diseño, implementación y monitoreo de las políticas y programas. (Chinchilla \& Vorndran, 2018). Estas informaciones son utilizadas por los sistemas Big Data para la prevención de la violencia y la delincuencia. (Vilalta-Perdomo, 2017). A pesar del uso de las TIC en la prevención del delito, se enfrenta el reto de la disponibilidad y calidad de la información. (Chinchilla \& Vorndran, 2018). Algunas informaciones no se encuentran disponibles, por ejemplo, ¿cuáles son los sectores donde existe mayor número de delitos?, ¿en cuáles lugares se están implementando los programas de intervención comunitaria?, ¿cuál es la metodología utilizada?, ¿quiénes participan?, ¿el costo de los programas?, ¿¿cuál es el seguimiento y control?, entre otras informaciones.

b. Utiliza el enfoque punitivo: más de 2,000 efectivos policiales y militares, quienes patrullaban día y noche las calles con el objetivo de proteger a la ciudadanía. La estrategia debe implementar un oficial en puntos calientes; se ha utilizado en los países de Chile, Colombia y Ecuador, entre otros países (Frühling, 2012). La vigilancia policial de los puntos calientes concentra la vigilancia policial en áreas de alta criminalidad o en delitos específicos como el tráfico de drogas. (Instituto de Políticas Públicas del Estado de Washington, WSIPP, 2017).

c. Estrategia para el control de drogas: se basa en la persecución, control del narcotráfico y microtráfico. No muestra estrategia para prevenir o disminuir el consumo de drogas, especialmente en los jóvenes. Por ejemplo, la estrategia "demasiado bueno para las drogas". (Instituto de Políticas Públicas del Estado de Washington, WSIPP, 2017).

d. Programa Vivir Tranquilo: se enfoca en la comunidad. Como se señaló anteriormente, las intervenciones basadas en la comunidad han tenido 


\section{Estrategias de intervención para disminuir la violencia y la delincuencia: avances y desafíos en República Dominicana}

efectos positivos en la diminución del delito y la violencia. De acuerdo con la información obtenida, el programa "Vivir Tranquilo" presenta las siguientes dificultades en su marco conceptual: problemas de Integridad: falta incorporación de los diversos organismos y sectores a la gestión de la seguridad, y la necesidad de diseñar estructuras organizativas que faciliten la coordinación y la cohesión interinstitucional. (Chinchilla \& Vorndran, 2018). Adicionalmente, dicho programa no integra los diferentes sectores de la sociedad en el diseño, ejecución y evaluación. Por otro lado, el plan no revela evidencias científicas de los resultados obtenidos de las intervenciones donde se ha implementado; es decir, se necesita evaluar el programa para verificar cuáles son los resultados. Los estudios experimentales o cuasi experimentales generan evidencia sobre el nivel de eficacia de una determinada intervención, en términos del nivel de logro de los objetivos con que se diseñó su modelo. (Tocornal et al., 2011).

e. Otras observacionesdel Plan Seguridad Ciudadana: no identifica la intervención en los tres niveles. Las políticas públicas deben estar basadas en prevenir la violencia y la delincuencia utilizando estrategias en los tres niveles. (Sistema de Integración Centroamericana, SICA, 2014). No establece estrategias para disminuir la reincidencia. En ese orden, se han identificado programas que han dado resultados para su disminución, por ejemplo: "Terapia familiar funcional (FFT) para jóvenes en instituciones estatales". Este programa tiene evidencia científica de que disminuye reincidencia. (Instituto de Políticas Públicas del Estado de Washington, WSIPP, 2017). También se han evaluado programas de terapia familiar para jóvenes; los cuales han mostrado evidencia de diminución de la reincidencia (Frühling, 2012).

El Plan de Seguridad Ciudadana no presenta estrategias para disminuir la sobrepoblación carcelaria. Según las estadísticas, la tasa de homicidios ha dis- minuido en los últimos años; $26 \%$ de 2000 a 2018; sin embargo, la sobrepoblación carcelaria aumentó un $22 \%$ desde 2008 . A esto se agrega que la condición jurídica de los privados de libertad, cerca del $65 \%$, es preventiva. Esta situación penitenciaria trae como consecuencias el hacinamiento, problemas de salud y un alto costo al Estado. En ese sentido, se debe mejorar la efectividad de la policía, optimizar el sistema de justicia penal y penitenciario. (Organización de Estados Americanos, OEA, 2012).

\section{Conclusiones}

Según las estadísticas, la tasa de homicidios alcanzó los niveles más altos durante la ejecución del Plan Seguridad Democrática, en el cual se implementó el "Programa Barrio Seguro".

El índice de homicidios disminuyó un $26 \%$ desde el año 2000 al 2018; sin embargo, la tasa de sobrepoblación penitenciaria aumentó un $22 \%$ desde 2008 a 2018.

Se ha evidenciado que las estrategias de Seguridad Ciudadana que ha realizado el Estado dominicano desde 2004 han dado buenos resultados en otros países.

Los programas de seguridad ciudadana en República Dominicana enfrentan los siguientes desafíos: integración de la sociedad en el diseño, ejecución y evaluación de políticas de seguridad ciudadana. Utilizar evidencias científicas para evaluar las estrategias. Diseñar programas de prevención terciaria. Disminuir la sobrepoblación carcelaria. Optimizar el sistema de justicia y el sistema penitenciario.

\section{Referencias}

Aguirre, K., \& Muggah, R. (2017). Las agencias multilaterales y el enfoque de «seguridad ciudadana» en América Latina. Revista CIDOB d'Afers Internacionals. doi: doi.org/10.24241/ rcai.2017.116.2.25. 
Barros Leal, C. (2014). La pena de prisión preventiva en América Latina: Los privados de libertad y sus derechos humanos. Revista Instituto Brasileño de Derechos Humanos. Recuperado de http://milas.x10host.com/ojs/index.php/ ibdh/article/view/265

Berk-Seligson, S., Orcés, D., Pizzolitto, G., Seligson, M. A., \& Wilson, C. J. (2014). Evaluación de impacto de la estrategia basada en la comunidad de USAID para la prevención de la delincuencia y la violencia en América Central: Informe regional para El Salvador, Guatemala, Honduras y Panamá. Proyecto de Opinión Pública de América Latina (LAPOP). Nashville: Vanderbilt University.

Carranza, E. (2012). Situación penitenciaria en AméricaLatinayel Caribe ¿Quéhacer? Anuario de Derechos Humanos 2012. Recuperado de https://anuariocdh.uchile.cl/index.php/ADH/ article/download/20551/21723/0

Chinchilla, L., \& Vorndran, D. (2018). Seguridad ciudadana en América Latina y el Caribe. Desafios e innovación en gestión y politicas públicas en los últimos 10 años. Programa de Estado de Derecho Peter D. Bell. Washington: Banco Interamericano de Desarrollo.

Comisión Interamericana de Derechos Humanos. (2011). Informe sobre los derechos humanos de las personas privadas de libertad en las Américas. $O E A$. Recuperado de https://www.oas.org/es/ cidh/ppl/docs/pdf/ppl2011esp.pdf

Comisión Interamericana de Derechos Humanos. (2017). Medidas para reducir la prisión preventiva. España: Organización de Estados Americanos.

Comisión Interamericana de Derechos Humanos, CIDH. (2009). Informe sobre seguridad ciudadana y derechos humanos. Organización de Estados Americanos.

Comisión Nacional de los Derechos Humanos. (2015). La sobrepoblación en los Centros Penitenciarios de la República Mexicana. México: Comisión Nacional de los Derechos Humanos.
Dirección de Información, Análisis y Programación Estratégica (DIAPE). (2016). Ejecuciones del Gobierno a favor de la seguridad ciudadana 2012-2016. República Dominicana: Presidencia de la República Dominicana.

Frühling, H. (2012). La eficacia de las politicas públicas de seguridad ciudadana en América Latina y el Caribe: cómo medirla y cómo mejorarla. Banco Interamericano de Desarrollo.

Instituto de Políticas Públicas del Estado de Washington, WSIPP. (2017). Resultados de costo-beneficio. Recuperado de Instituto de Políticas Públicas del Estado de Washington.

Instituto Interamericano de Derechos Humanos, IIDH. (2011). Derechos humanos, seguridad ciudadana y funciones policiales. San José, Costa Rica: Editorial-Servicios Especiales del IIDH.

INTERPEACE. (2011). Estrategias para prevenir la violencia juvenil. Ciudad de Guatemala: INTERPEACE.

Izquierdo, A., Pessino, C., \& Vuletin, G. (2018). Mejor gasto para mejores vidas. Cómo América Latina y el Caribe puede hacer más con menos. Banco Interamericano de Desarrollo.

Latinobarómetro. (2016). Informe 2016. Buenos Aires: Banco Interamericano de Desarrollo.

Oficina de las Naciones Unidas contra la Droga y el Delito, UNODC. (2014). Manual Sobre Estrategias Para Reducir el Hacinamiento en las Prisiones. New York: UNODC.

Oficina de las Naciones Unidas Contra la Droga y el Delito, UNODC. (2011). Manual de instrucciones para la evaluación de la justicia penal. New York: UNODC.

Oficina Nacional de Estadística. (2016). Encuesta Nacional de Hogares de Propósitos Múltiples (ENHOGAR). Santo Domingo: Oficina Nacional de Estadística.

Organización de Estados Americanos, OEA. (2012). Informe sobre Seguridad Ciudadana en las Américas 2012. Organización de Estados Americanos. 
Organización Mundial de la Salud, OMS. (2014). InformeSobrelaSituaciónMundialdelaPrevención de la Violencia. Washington, D.C.: Organización Mundial de la Salud. Recuperado de apps.who. int/iris/bitstream/10665/145089/1/WHO_ NMH_NVI_14.2_spa.pdf

Organización Panamericana de la Salud. (2016). La prevención de la violencia juvenil: panorama general de la evidencia. Washington, D.C.: Organización Mundial de la Salud.

Ortiz Rodríguez, M. (2018). Propuesta de un proyecto para disminuir los altos índices de violencia y delincuencia en la provincia de La Romana, República Dominicana. Ciencia y Sociedad, 43(3). doi: http://dx.doi. org/10.22206/cys.2018.v43i3.pp41-55

Procuraduría General de la República, PGR. (2016). Informe de Homicidios - Enero Diciembre 2015. Fuente Policía Nacional. Santo Domingo: Procuraduría General de La República. Recuperado de http:// estadisticas.pgr.gob.do/documentos/ homicidios/20160208-informe-homicidios-ene-dic-2014-2015.ashx

Programa de las Naciones Unidas para el Desarrollo, PNUD. (2014). Informe Regional de Desarrollo Humano 2013-2014. Seguridad Ciudadana con rostro humano. Diagnóstico y propuesta para América Latina. Panamá: Alfa Omega Impresiones.
Seguridad, Justicia y Paz. (2018). Metodología del ranking (2017) de las 50 ciudades más violentas del mundo. México. Recuperado de https://www.seguridadjusticiaypaz.org.mx/ biblioteca/prensa/category/6-prensa

Seguridad, Justicia y Paz. (2019). Metodología del ranking (2018) de las 50 ciudades más violentas del mundo. México: CCSPJP AC.

Sistema de Integración Centroamericana, SICA. (2014). Marco Estratégico para la prevención de la violencia. Panamá: Secretaría General del SICA.

Thornton, T., Craft, C. A., Dahlberg, L. L., Lynch, B. S., \& Baer, K. (2001). Prácticas óptimas para la prevención de la violencia juvenil. Atlanta: Department of Health and Human Services. Recuperado de https://www.cdc.gov/ violenceprevention/pdf/bestpracticesspan.pdf

Tocornal, X., Blanco, J., Varela, J., Llorente, M. V., Bulla, P., Castillo, J., . . W Wigodzky, V. (2011). Experiencias en América Latina: el desafio de evaluar programas de seguridad ciudadana. Chile: Centro de Estudios en Seguridad Ciudadana.

United Nations Office on Drugs and Crime, (UNODC). (2019). Global study on homicide. Viena: United Nations Office on Drugs and Crime.

Vilalta-Perdomo, C. J. (2017). Información para la prevención del delito y la violencia. Quito: Banco Interamericano de Desarrollo.

\section{Datos de filiación}

Candidata a doctora en Proyectos, Universidad Internacional Iberoamericana UNINI México. Línea de investigación: Proyectos Integrados. Tiene una maestría Psicología Clínica, por la Universidad UTESA. Cursos y especialidades: Evaluación de Impacto de Proyectos Sociales, J-PAL; Técnicas Cuantitativas y Cualitativas de la Investigación, Universidad Politécnica de Valencia; gestión de Proyectos de Desarrollo, Banco Interamericano de Desarrollo; aspirante a Trabajadora Social, Escuela Nacional de la Judicatura; Intervención Social en protección al menor, Universidad Ryerson, Canadá, y la Facultad de Ciencias Económicas y Sociales de la Universidad Autónoma de Santo Domingo. 